ESAIM: PROCEEDINGS, March 2012, Vol. 35, p. 245-250

Fédération Denis Poisson (Orléans-Tours) et E. Trélat (UPMC), Editors

\title{
A COUPLED WELL-BALANCED AND RANDOM SAMPLING SCHEME FOR COMPUTING BUBBLE OSCILLATIONS*
}

\author{
Philippe Helluy ${ }^{1}$ and Jonathan Jung ${ }^{2}$
}

\begin{abstract}
We propose a finite volume scheme to study the oscillations of a spherical bubble of gas in a liquid phase. Spherical symmetry implies a geometric source term in the Euler equations. Our scheme satisfies the well-balanced property. It is based on the VFRoe approach. In order to avoid spurious pressure oscillations, the well-balanced approach is coupled with an ALE (Arbitrary Lagrangian Eulerian) technique at the interface and a random sampling remap.

Résumé. Nous proposons un schéma de volumes finis pour étudier les oscillations d'une bulle sphérique de gaz dans l'eau. La symétrie sphérique fait apparaitre un terme source géométrique dans les équations d'Euler. Notre schéma est basé sur une approche VFRoe et préserve les états stationnaires. Pour éviter les oscillations de pression, l'approche well-balanced est couplée avec une approche ALE (Arbitrary Lagrangian Eulerian), et une étape de projection basée sur un échantillonage aléatoire.
\end{abstract}

\section{INTRODUCTION}

We want study the behavior of a spherical bubble of gas in a liquid phase. Note that the equations will be the same as in the case of flows in variable cross-section. Classical finite volume solvers generally have a bad precision for solving two-fluid interfaces or flows in varying cross-section ducts. Several cures have been developed for improving the precision.

- For cross-section ducts, the well-balanced approach of Greenberg and Leroux [5] (see also $[6,10]$ ) is an efficient tool to improve the precision.

- For two-fluid flows the pressure oscillations phenomenon (see $[2,8]$ for instance) can be cured by a recent tool developed in $[1,4]$. It is based on an ALE (Arbitrary Lagrangian Eulerian) scheme followed by a random sampling projection step.

In this paper, we show that it is possible to mix the two approaches in order to design an efficient scheme for computing the collapse of a spherical bubble of gas in a liquid phase.

\section{MOdel}

\subsection{Equations}

To study a spherical bubble of gas in a liquid phase we consider the 3D-Euler equations. We assume that the flow is invariant under rotation. We note $x$ the space variable along the radius of the bubble and $t$ the time (see

* This work has been partly supported by a PICS CNRS grant.

${ }^{1}$ IRMA, University of Strasbourg; helluy@math.unistra.fr

2 IRMA, University of Strasbourg; jonathan.jung@math.unistra.fr. 
Figure 1(a)). With the spherical symmetry the unknowns are the density $\rho$, the radial velocity $u$, the internal energy $e$ and the fraction of gas $\varphi$ and they depend only on $(x, t)$. We can put the 3D-Euler equations in the following form

$$
\begin{aligned}
\partial_{t}(A \rho)+\partial_{x}(A \rho u) & =0, \\
\partial_{t}(A \rho u)+\partial_{x}\left(A\left(\rho u^{2}+p\right)\right) & =p \partial_{x} A, \\
\partial_{t}(A \rho E)+\partial_{x}(A(\rho E+p) u) & =0, \\
\partial_{t}(A \rho \varphi)+\partial_{x}(A \rho \varphi u) & =0, \\
\partial_{t} A & =0,
\end{aligned}
$$

where

$$
p=p(\rho, e, \varphi), \quad E=e+\frac{u^{2}}{2},
$$

and $A(x)=x^{2}$ appears because of spherical symmetry. Without loss of generality, in this paper we consider a stiffened gas pressure law (see [11] and included references)

$$
p(\rho, e, \varphi)=(\gamma(\varphi)-1) \rho e-\gamma(\varphi) \pi(\varphi),
$$

where $(\gamma, \pi)(\varphi=1)=\left(\gamma_{g a s}, \pi_{g a s}\right)$ and $(\gamma, \pi)(\varphi=0)=\left(\gamma_{l i q}, \pi_{l i q}\right)$.

We can also put the system (1)-(5) in the non-conservative form

$$
\partial_{t} W+\partial_{x} F(W)-S(W) \partial_{x} A=0,
$$

where

$$
\begin{aligned}
W & =(A \rho, A \rho u, A \rho E, A \rho \varphi, A)^{T} \\
F(W) & =\left(A \rho u, A\left(\rho u^{2}+p\right), A(\rho E+p) u, A \rho \varphi u, 0\right)^{T}, \\
S \partial_{x} A & =\left(0, p \partial_{x} A, 0,0,0\right) .
\end{aligned}
$$

We define the vector of primitive variables

$$
Y=(\rho, u, p, \varphi, A)^{T} .
$$

We define also the following quantities

$$
\begin{aligned}
Q=\text { mass flow rate }=\rho A u, & s=\text { entropy }=(p+\pi(\varphi)) \rho^{-\gamma(\varphi)}, \\
h=\text { enthalpy }=e+\frac{p}{\rho}, & H=\text { total enthalpy }=h+\frac{u^{2}}{2} .
\end{aligned}
$$

The sound speed $c$ satisfies

$$
c^{2}=p_{\rho}=\rho h_{\rho}
$$

\subsection{Properties}

Proposition 1.1. The jacobian matrix of the non conservative system (8) admits five real eigenvalues

$$
\lambda_{0}=0, \quad \lambda_{1}=u-c, \quad \lambda_{2}=\lambda_{3}=u, \quad \lambda_{4}=u+c .
$$

However, the system may be resonant (when $\lambda_{0}=\lambda_{1}$ or $\lambda_{0}=\lambda_{4}$.)

The quantities $\varphi, s, Q$ and $H$ are independent Riemann invariants of the stationary wave $\lambda_{0}$.

In the sequel, the vector of "stationary" variables $Z=(A, \varphi, s, Q, H)^{T}$ will play a particular role. 


\section{A WELL-BALANCED TWO-FLUID ALE SOLVER}

\subsection{Principe}

The objective of our algorithm is to determine the behavior of a spherical gas bubble. For computing the gasliquid interface, we propose to mix front-tracking and front-capturing approaches. First, we apply Arbitrary Lagrangian Eulerian (ALE) ideas: at the interface between gas and water we use a Lagrangian approach. Elsewhere, we use an Eulerian scheme.

We divide the domain $\Omega$ into $N$ cells $\left.\Omega_{i}=\right] x_{i-1 / 2}, x_{i+1 / 2}$ [ centered on $x_{i}$. We denote by $\tau$ the time step and by $\Delta x_{i}=x_{i+1 / 2}-x_{i-1 / 2}$ the size of cell $i$. The area of the section $A$ is approximated by a piecewise constant function, $A=A_{i}$ in cell $\Omega_{i}$.

The boundary $x_{i+1 / 2}^{n}$ can move at the velocity of the fluid $v_{i+1 / 2}^{n}$ between time $t_{n}$ and $t_{n+1}$, thus we have

$$
x_{i+1 / 2}^{n+1,-}=x_{i+1 / 2}+\tau v_{i+1 / 2}^{n}
$$

where at the gas-liquid interface (i.e $\varphi_{i}^{n} \neq \varphi_{i+1}^{n}$ ) we have $v_{i+1 / 2}^{n} \neq 0$, everywhere else we have $v_{i+1 / 2}^{n}=0$.

Suppose that at time $t_{n}$ we know an approximation $W^{n}$ of the exact solution $W$. The approximation is constant on each cell

$$
W\left(t_{n}, x\right) \simeq W^{n}(x)=W_{i}^{n}, \quad x \in \Omega_{i} .
$$

As $A$ is constant on each cell, the integration of the non-conservative system (8) on the quadrilateral space-time

$$
\begin{aligned}
Q & =\left\{(x, t) \in \mathbb{R}^{2} \mid t_{n}<t<t_{n+1}, x_{i-\frac{1}{2}}^{n}+t \times v_{i-1 / 2}^{n}<x<x_{i+\frac{1}{2}}^{n}+t \times v_{i+1 / 2}^{n}\right\} \\
& \left.=\bigcup_{t_{n}<t<t_{n+1}}\right] x_{i-\frac{1}{2}}^{n}+t \times v_{i-1 / 2}^{n} ; x_{i+\frac{1}{2}}^{n}+t \times v_{i+1 / 2}^{n}[\times\{t\}
\end{aligned}
$$

gives

$$
\iint_{Q} \partial_{t} W+\partial_{x} F(W)=0
$$

To simplify the formula, we define a discontinuous Arbitrary Lagrangian Eulerian (ALE) numerical flux

$$
\left.F\left(W_{L}, W_{R}, v^{ \pm}\right):=F\left(W\left(W_{L}, W_{R}, v^{ \pm}\right)\right)-v W\left(W_{L}, W_{R}, v^{ \pm}\right)\right)
$$

where $W\left(W_{L}, W_{R}, v^{ \pm}\right)=W^{\text {Riemann }}\left(W_{L}, W_{R}, \frac{x}{t}=v^{ \pm}\right)$. We cannot confuse the fluxes denoted by $F$ because they do not depend on the same number of variables.

By applying Stokes' formula to (19), we have

- if $v_{i+1 / 2}^{n} \leq 0$ and $v_{i-1 / 2}^{n} \geq 0$

$$
\Delta x_{i}^{n+1,-} W_{i}^{n+1,-}-\Delta x_{i} W_{i}^{n}+\tau\left(F\left(W_{i}^{n}, W_{i+1}^{n}, v_{i+1 / 2}^{n,-}\right)-F\left(W_{i-1}^{n}, W_{i}^{n}, v_{i-1 / 2}^{n,+}\right)\right)=0,
$$

- In order to take into account the variable section, we also have to make the following corrections

- if $v_{i+1 / 2}^{n}>0$, we add the following term on the left side of the equation above

$$
\tau\left(F\left(W_{i}^{n}, W_{\text {moy }}^{n}, 0^{-}\right)-F\left(W_{i}^{n}, W_{\text {moy }}^{n}, 0^{+}\right)\right),
$$

where $W_{\text {moy }}^{n}=W\left(Y_{\text {moy }}^{n}=\left(\rho_{i}^{n}, u_{i}^{n}, p_{i}^{n}, \varphi_{i}^{n}, A_{i+1}^{n}\right)\right)$,

- if $v_{i-1 / 2}^{n}<0$, we add

$$
\tau\left(F\left(W_{\text {moy }}^{n}, W_{i}^{n}, 0^{-}\right)-F\left(W_{\text {moy }}^{n}, W_{i}^{n}, 0^{+}\right)\right) .
$$

where $W_{\text {moy }}^{n}=W\left(Y_{\text {moy }}^{n}=\left(\rho_{i}^{n}, u_{i}^{n}, p_{i}^{n}, \varphi_{i}^{n}, A_{i-1}^{n}\right)\right)$. 
In this equality, the time step $\tau$ satisfies a CFL condition

$$
\tau \leq \max _{i} \frac{\triangle x_{i}}{\left|u_{i}\right|+c_{i}}
$$

To update $W_{i}^{n+1,-}$, we have to compute $F\left(W_{L}, W_{R}, v^{ \pm}\right)$. To do that, we need to:

- precise how we choose the ALE velocity $v$,

- explain how we compute $F\left(W_{L}, W_{R}, v^{ \pm}\right)$for a given ALE velocity $v$.

\subsection{ALE velocity}

We have now to express the velocity $v$ that depends on the data $W_{L}$ and $W_{R}$. The idea is to use the well-balanced scheme everywhere except at the interface between the two fluids. At this interface we use the Lagrange flux. When our initial data satisfy $\varphi \in\{0,1\}$, the algorithm reads:

- if we are not at the interface, i.e. if $\varphi_{L}=\varphi_{R}$, we take $v=0$,

- if we are at the interface, i.e. if $\varphi_{L} \neq \varphi_{R}$. We use an exact Riemann solver of variable $(\rho, u, p, \varphi)$ to compute the pressure and the velocity at the contact discontinuity, which presents no jump at this discontinuity. We thus denote by $u^{*}\left(W_{L}, W_{R}\right)$ and $p^{*}\left(W_{L}, W_{R}\right)$ the velocity and the pressure at the contact. We take

$$
\begin{aligned}
& v=u^{*}\left(W_{L}, W_{R}\right),
\end{aligned}
$$

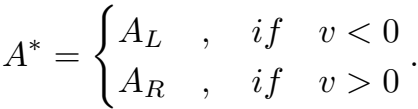

The numerical flux ALE becomes lagrangian and takes the form

$$
F\left(W_{L}, W_{R}, v^{ \pm}\right)=\left(0, A^{*} p^{*}, A^{*} u^{*} p^{*}, 0,-A^{*} u^{*}\right)^{T} .
$$

\subsection{Computing $W\left(W_{L}, W_{R}, 0^{ \pm}\right)$}

As the invariants of Riemann associated to the stationary wave are $\varphi, s, Q, H$ (prop 1.1), the idea is to use a VFRoe ncv solver (see [3]) in the variables $Z=(A, \varphi, s, Q, H)$ to compute the interface states $W\left(W_{L}, W_{R}, 0^{ \pm}\right)$. We proceed as follows. For regular solutions, system (1)-(5) may be rewritten in the non-conservative form

$$
\partial_{t} Z+C(Z) \partial_{x} Z=0
$$

where

that admits 5 real eigenvalues

$$
C(Z)=\left[\begin{array}{ccccc}
0 & 0 & 0 & 0 & 0 \\
0 & u & 0 & 0 & 0 \\
0 & 0 & u & 0 & 0 \\
0 & A\left(\partial_{\varphi} p-\rho \partial_{\varphi} h\right) & A\left(\partial_{s} p-\rho \partial_{s} h\right) & u & \rho A \\
0 & \frac{u}{\rho} \partial_{\varphi} p & \frac{u}{\rho} \partial_{s} p & \frac{c^{2}}{\rho A} & u
\end{array}\right]
$$

$$
\lambda_{0}=0, \quad \lambda_{1}=u-c, \quad \lambda_{2}=\lambda_{3}=u, \quad \lambda_{4}=u+c .
$$

Now, we want to solve

with the initial data

$$
\partial_{t} Z+C(Z) \partial_{x} Z=0
$$

$$
Z(x, 0)= \begin{cases}Z\left(W_{L}\right) & , x<0 \\ Z\left(W_{R}\right) & , x>0\end{cases}
$$


- We approximate $C(Z)$ at $\hat{Z}=Z\left(\frac{Y_{L}+Y_{R}}{2}\right)$ and we solve the linear Riemann problem

$$
\partial_{t} Z+C(\hat{Z}) \partial_{x} Z=0
$$

with the same initial datas.

- We obtain $Z\left(W_{L}, W_{R}, 0^{ \pm}\right)$.

- We go back to $W\left(W_{L}, W_{R}, 0^{ \pm}\right)$. It appears that this change of variable is not always invertible. We deal with this difficulty as in [6].

The VFRoe ncv scheme without entropy correction can approach incorrect rarefaction waves crossing the interface $x=0$ and thus authorizes in these points non physical shocks. To solve this problem, we use the following entropy correction presented in [7]:

Definition 2.1. If $\lambda_{k}\left(W_{i}^{n}\right) \leq 0 \leq \lambda_{k}\left(W_{i+1}^{n}\right)$ in a non linear k-field, then the numerical flux $F$ is replaced by

$$
\begin{aligned}
G\left(W_{i}^{n}, W_{i+1}^{n}, 0^{ \pm}\right) & =F\left(W_{i}^{n}, W_{i+1}^{n}, 0^{ \pm}\right) \\
& -\min _{k}\left(\left|\lambda_{k}\left(W_{i}^{n}\right)\right|,\left|\lambda_{k}\left(W_{i+1}^{n}\right)\right|\right)\left(W_{i+1}^{n}-W_{i}^{n}\right) / 2 .
\end{aligned}
$$

\subsection{Glimm remap}

We go back to the original Euler grid by the Glimm procedure. We construct a sequence of pseudo-random numbers $\omega_{n} \in[0,1[$. In practice, we consider the $(5,3)$ van der Corput sequence [1]. According to this number we take

$$
W_{i}^{n+1}= \begin{cases}W_{i-1}^{n+1,-}, & \text { if } \omega_{n}<\frac{\tau}{\Delta x_{i}} \max \left(v_{i-1 / 2}^{n}, 0\right), \\ W_{i}^{n+1,-}, & \text { if } \frac{\tau}{\Delta x_{i}} \max \left(v_{i-1 / 2}^{n}, 0\right) \leq \omega_{n} \leq 1+\frac{\tau}{\Delta x_{i}} \min \left(v_{i+1 / 2}^{n}, 0\right), \\ W_{i+1}^{n+1,-}, & \text { if } \omega_{n}>1+\frac{\tau}{\Delta x_{i}} \min \left(v_{i+1 / 2}^{n}, 0\right) .\end{cases}
$$

\subsection{Properties of the scheme}

The constructed scheme has many interesting properties:

- it is well-balanced in the sense that it preserves exactly all stationary states (i.e. initial data for which the quantities $\varphi, s, Q, H$ are constant);

- for a flow in a duct with constant cross-section ducts, it computes exactly the contact discontinuities, with no smearing at the interface;

- if at the initial time the mass fraction is in $\{0,1\}$, then this property is exactly preserved at any time.

For detailed proofs, we refer to $[1,6]$. Some other subtleties are given in the same references. For instance, the change of variables $Z=Z(W)$ is not always invertible, that implies to define a special procedure for constructing completely rigorously the well-balanced VFRoe solver.

\section{Numerical Result: Implosion of A BUbBle}

We simulate the collapse of a spherical bubble of vapor in liquid water. In this case we recall that $A(x)=x^{2}$. We assume that the initial radius of the bubble is $R_{\max }=0,7469 \times 10^{-4}$. The computation is carried out on $\Omega=\left[0 ; 20 \times R_{\max }\right]$ with approximatively $N=15000$ cells. Initial datas are the following:

\begin{tabular}{|c|c|c|}
\hline Quantity & Left & Right \\
\hline$\rho\left(\mathrm{kg} \cdot \mathrm{m}^{-3}\right)$ & 0.92 & 1000 \\
\hline$u\left(\mathrm{~m} \cdot \mathrm{s}^{-1}\right)$ & 0 & 0 \\
\hline$p(P a)$ & 72567.68 & $10^{5}$ \\
\hline$\varphi$ & 1 & 0 \\
\hline$\gamma$ & 1.4 & 3 \\
\hline$\pi(P a)$ & 0 & 733333.33 \\
\hline
\end{tabular}




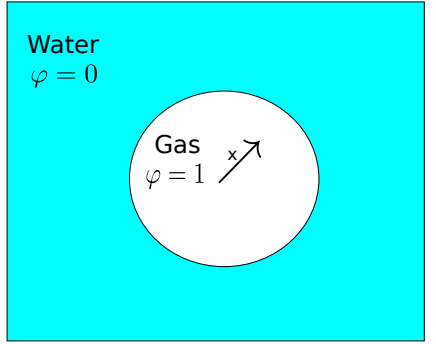

(a) Illustration of the problem

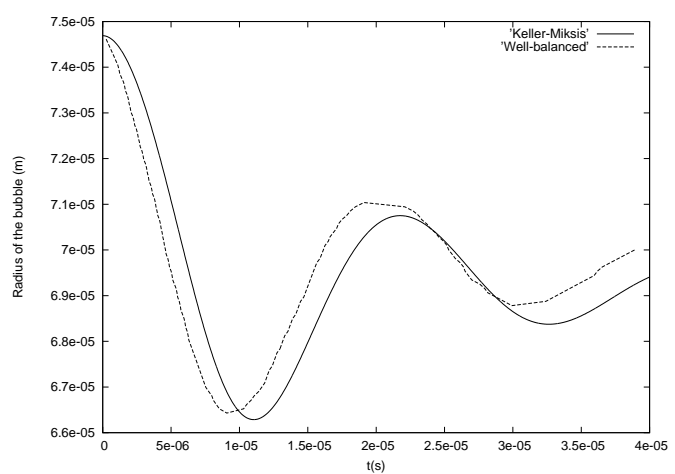

(b) Test of the bubble: oscillation of the radius of the bubble

\section{FIGURE 1}

The amplitude of the oscillations of the radius obtained with our scheme (see Figure 1(b)) is comparable with those obtained with the model of Keller-Miksis (see [9]). Let us remind that the model of Keller-Miksis is an estimate of the real flow. It must be considered as a reference solution but not as an exact solution.

\section{Conclusion}

We have constructed and validated a new one order scheme to compute the oscillation of a spherical bubble in a liquid phase. The smooth function $A$ is approximated by a piecewise constant function. Our scheme relies on two ingredients:

- a well-balanced approach for dealing with the varying cross-section;

- a Lagrange plus remap technique in order to avoid pressure oscillations at the interface. The random sampling remap ensures that the interface is not diffused at all.

Our scheme can also be applied to flows in a nozzle with variable cross-section.

\section{REFERENCES}

[1] M. Bachmann, P. Helluy, H. Mathis, S. Mueller, Random sampling remap for compressible two-phase flows, Preprint HAL http://hal.archives-ouvertes.fr/hal-00546919/fr/.

[2] T. Barberon, P. Helluy, S. Rouy, Practical computation of axisymmetrical multifluid flows, Int. J. Finite Vol. 1, no. 1, 34 pp, 2004.

[3] T. Buffard, T. Gallouet, J.-M. Hérard. A sequel to a rough Godunov scheme: application to real gases, Computers and Fluids, vol. 29, pp. 813-847, 2000.

[4] C. Chalons, F. Coquel. Computing material fronts with a Lagrange-Projection approach. HYP2010 Proc. http://hal.archivesouvertes.fr/hal-00548938/fr/.

[5] J.-M. Greenberg, A.Y., Leroux. A well balanced scheme for the numerical processing of source terms in hyperbolic equations, SIAM J. Num. Anal., vol. 33 (1), pp. 1-16, 1996.

[6] P. Helluy, J.-M. Hérard, H. Mathis. A Well- Balanced Approximate Riemann Solver for Variable Cross- Section Compressible Flows. AIAA-2009-3540. 19th AIAA Computational Fluid Dynamics. June 2009.

[7] P. Helluy, J.-M Hérard, H. Mathis, S. Mueller. A simple parameter-free entropy correction for approximate Riemann solvers, Comptes rendus Mécanique, 2010.

[8] S. Karni. Multicomponent flow calculations by a consistent primitive algorithm. J. Comput. Phys. 112, no. 1, pp. 31-43, 1994.

[9] B. Keller, M.Miksis. Bubble oscillations of large amplitude, J. Acoust. Soc. Am., 68(2), pp. 628-633, 1980.

[10] D. Kroner, M.-D. Thanh. Numerical solution to compressible flows in a nozzle with variable cross-section, SIAM J. Numer. Anal., vol. 43(2), pp. 796-824, 2006.

[11] R. Saurel, R. Abgrall. A simple method for compressible multifluid flows, SIAM J. Sci. Comput. 21, no. 3, pp. 1115-11145, 1999. 